Clément Makiobo, Église catholique et mutations sociopolitiques au Congo-Zaïre.La contestation du régime de Mobutu

Paris, L'Harmattan, coll. « Congo/Zaïre Histoire \& Société », 2004, 264 p.

Joseph Tonda

\title{
OpenEdition
}

Journals

Édition électronique

URL : http://journals.openedition.org/assr/3569

DOI : 10.4000/assr.3569

ISSN : $1777-5825$

Éditeur

Éditions de l'EHESS

Édition imprimée

Date de publication : 1 mai 2006

Pagination : 147-299

ISBN : 2-7132-2092-0

ISSN : 0335-5985

Référence électronique

Joseph Tonda, « Clément Makiobo, Église catholique et mutations socio-politiques au Congo-Zairre.La contestation du régime de Mobutu », Archives de sciences sociales des religions [En ligne], 134 | avril - juin 2006, document 134-56, mis en ligne le 11 septembre 2006, consulté le 21 septembre 2020. URL : http://journals.openedition.org/assr/3569 ; DOI : https://doi.org/10.4000/assr.3569 


\section{Clément Makiobo, Église catholique et mutations socio-politiques au Congo- Zaïre.La contestation du régime de Mobutu}

Paris, L'Harmattan, coll. « Congo/Zaïre Histoire \& Société », 2004, 264 p. Joseph Tonda

L'objet principal du livre de Clément Makiobo est l'analyse des textes produits par l'épiscopat catholique du Congo/Zaïre entre 1972 et 1997 : textes dont les thèmes renseignent sur les orientations fondamentales de l'Église catholique confrontée à la dictature de Mobutu. En effet, c'est entre 1972 et 1997 que s'instaure, s'intensifie et meurt l'idéologie « laïque » qui va diviniser Mobutu, en l'affublant des noms de « Père de la nation », "Grand Timonier », « Héros national », « Président-fondateur du MPR, Mouvement populaire de la révolution », «Guide suprême de la révolution zaïroise ». C'est dans un document publié en 1967, intitulé le «Manifeste de la N'Selé» que se formule pour la première fois le «nationalisme congolais authentique » qui devait conduire à l'« authenticité zaïroise ». Cette " authenticité », entendue comme quête de l'«identité africaine » et proclamée comme philosophie politique, est théorisée, comme dans le cas de l'ivoirité, par des intellectuels. D'après Mobutu lui-même, cette philosophie procède d'une "prise de conscience du peuple zaïrois de recourir à ses sources propres, de rechercher des valeurs de ses ancêtres afin d'en apprécier celles qui contribuent à son développement harmonique et naturel [...]. C'est l'affirmation de l'homme tout court, là où il est tel qu'il est, avec ses structures mentales et sociales propres» (p. 90). Les textes analysés par Clément Makiobo sont donc des prises de position de l'Église du Congo contre cette idéologie. Mais l'auteur s'est aussi intéressé aux mouvements d'action catholique nés au Zaïre après 1972 et qui ont en commun d'opérer un « changement individuel et collectif afin de rendre plus authentique et plus évangélique le christianisme local » (p.4). 
2 Le livre de $\mathrm{Cl}$. Makiobo ne raconte pas une histoire du combat de l'Église catholique contre la politique de Mobutu sur un terrain historique "vierge ». Les rapports entre l'Église et l'État postcolonial, dans la période 1972 à 1997 au Congo, s'inscrivent dans une histoire qui commence avec la colonisation au xix ${ }^{e}$ siècle. Cette histoire coloniale est celle de la collaboration des missions catholiques au Congo avec l'administration coloniale. Elle est aussi celle des œuvres et des mouvements de jeunesse catholiques qui ont fécondé des mouvements religieux congolais. Qu'apportent les missions catholiques au Congo? Clément Makiobo nous rappelle que c'est dans le cadre de l'État indépendant du Congo, propriété du roi des Belges Léopold II, et ensuite dans la colonie belge, que travaillent les missions. Entre 1880 et 1940, vingt-deux institutions de prêtres missionnaires, six congrégations de frères et cinquante-deux congrégations féminines sont dénombrées. Elles évangélisent et assurent l'instruction des congolais sur toute l'étendue du territoire. Les missionnaires sont, à cet effet, une composante du dispositif colonial du roi des Belges qui comprend l'Église catholique, les sociétés coloniales, l'administration et l'armée. C'est donc sous la protection du pouvoir colonial que les missionnaires livrent la guerre contre la «sauvagerie ", le "paganisme » et l'«animisme » des Noirs. Cette guerre, ils vont la livrer à travers la scolarisation, les œuvres sanitaires et les « villages chrétiens ».

3 L'auteur nous apprend que, jusqu'en 1946 au moins, c'est avec les subsides de l'État, que l'Église catholique s'est trouvée en situation dominante au Congo en matière d'enseignement. Cette école, rappelle-t-il, est moins destinée à une formation intellectuelle des autochtones qu'à l'apprentissage d'un métier manuel, car il n'est pas question d'encourager la «vanité » du Noir qui, par l'instruction, « devient facilement un facteur d'influence dissolvante ", "critique les Européens, excite les Noirs contre eux en exagérant les défauts de ceux-ci et les griefs de ceux-là, bref, [...] devient une nuisance et un danger. Il faut donc qu'il travaille, et c'est à la moralisation du Nègre par le travail, à savoir par le travail manuel, que doit tendre l'enseignement que nous lui donnons » (p. 24).

4 Le système des soins mis en place par les missionnaires est l'autre moyen de « civiliser». Cl. Makiobo souligne que la " plupart des grands hôpitaux et dispensaires de brousse étaient gérés par les missions catholiques grâce aux subsides de l'État et à la compétence de nombreuses congrégations religieuses [...]. La veille de l'indépendance, l'infrastructure sanitaire du Congo était certes insuffisante mais elle était efficace. En 1966, le Congo comptait 31 hôpitaux avec une capacité de 8000 lits, 59 cliniques, 391 maternités, 3881 centres paramédicaux ou dispensaires, 86 léproseries, 8 sanatoriums et 4 hôpitaux psychiatriques. L'Église catholique contrôlait à elle seule 60 \% de cette infrastructure » (p. 24).

5 Conçus comme un véritable lieu expérimental de « civilisation » et donc de conversion, les villages chrétiens répondaient à la nécessité d'arracher les Congolais au milieu ambiant du « paganisme " pour leur enseigner le catéchisme, la lecture et l'écriture, la prière et le chant. Fonctionnant non seulement comme dispositif de surveillance de la conduite des catéchumènes et chrétiens, en empêchant le "fétichisme", les "danses obscènes ", mais également comme moyen pour engager les habitants à se convertir, ces villages devaient également " contribuer à améliorer le sort temporel, la richesse et la variété des cultures et des plantations ; en général, aider le Père à gagner des âmes et des villages à Jésus-Christ et à procurer à leurs frères les bienfaits de la civilisation chrétienne». 
6 Les mouvements de jeunesse voient le jour, à l'initiative des missionnaires, pour résoudre les problèmes inhérents à l'urbanisation, l'alcoolisme, le banditisme, la prostitution. Par ailleurs, du fait de la fermeture de l'espace politique aux Congolais, l'expression politique des dominés congolais va prendre la forme des mouvements messianiques, dont le Kimbanguisme et le Kitawala.

7 C'est dans le cadre et le terreau de cette histoire des rapports entre l'Église et le pouvoir colonial que l'auteur montre la complexité des thèmes sur lesquels l'Église du Congo et le régime de Mobutu vont se confronter dans l'ère postcoloniale. Sur le plan idéologique, le thème central de cette confrontation est l'« authenticité ». Si l'Église catholique définit sa mission comme celle "d'aider l'Africain à ne pas se renier luimême, mais à se renouveler dans le Christ sous qui tout doit être ramené $(E p h .1,10)$ » et qu'elle "encourage les catholiques zaïrois et tous les Zaïrois de bonne volonté à conserver en eux le sens et le respect du sacré » (p. 104), ce texte ne s'en intitule pas moins «le christianisme source de l'authenticité». C'est donc le sens de l'« authenticité » qui est ici l'enjeu de la confrontation. Par ailleurs, cette confrontation se fait sur une sorte de chassé-croisé entre deux hommes qui ont fortement marqué la période historique étudiée dans le livre de $\mathrm{Cl}$. Makiobo, parce qu'ils incarnent les deux pouvoirs qui dominent le champ du pouvoir au Zaïre : Malula et Mobutu.

Éduqués tous deux par les pères belges de Scheut, ces deux hommes seront travaillés par le souci de réaliser l'hégémonie de leur pouvoir, mais en s'appuyant sur un usage stratégique de la «tradition» ou de l'« authenticité africaine». Arrivé au pouvoir avec l'aide des États-Unis d'Amérique, de la Belgique et de la France soucieux de ne pas voir le Congo basculer dans le camp communiste, Mobutu, né le 14 octobre 1930 dans la province de l'Équateur, a été éduqué par les prêtres de Scheut qui l'ont fait s'enrôler dans l'armée coloniale, la Force publique d'où il sort avec le grade de sergent en 1956 : il s'agit du grade le plus élevé qu'un Congolais pouvait obtenir dans l'armée belge. Puis Mobutu devient journaliste. Ami de Patrice Lumumba, c'est ce dernier qui l'amène au pouvoir en le nommant colonel afin de rétablir l'ordre dans l'armée qui connaît des troubles dans ces premiers temps de l'indépendance. On connait la suite: Mobutu prend le pouvoir en 1965 et va incarner, successivement, l'espoir du peuple congolais de 1965 à 1973, et la mégalomanie de 1973 à 1990. Malula, né le 17 décembre 1917 à Léopoldville (Kinshasa) dans une famille chrétienne fait ses études primaires dans une école dirigée par les pères de Scheut, avant d'aller faire ses humanités, de 1931 à 1934, au petit séminaire de Mbata Kiela, sur un territoire ecclésiastique confié aux mêmes pères. Il suit une formation de trois ans en philosophie et de cinq ans en théologie et, le 9 juin 1946, est ordonné prêtre à Léopoldville. Après un parcours particulièrement brillant, Malula est élevé au rang de cardinal le 28 mars 1969. Si Mobutu est divinisé à travers les nombreux pseudonymes attribués par ses chantres, Malula est, aussi, qualifié de «Père de l'inculturation » (p. 85), parce qu'« il prôna sans cesse l'intégration des valeurs culturelles africaines dans le christianisme et la christianisation de toute la vie politique, sociale et économique » (p. 85) et de "Père de l'Église de Kinshasa ». Cette guerre des "pères ", que se livrent Mobutu et Malula, se situe sur le terrain religieux, comme le montre le "Manifeste de la N'selé ", qualifié de "Catéchisme », et le MPR proclamé "Église». C'est pour imposer l'hégémonie religieuse de son pouvoir que Mobutu supprime les noms et prénoms chrétiens d'origine étrangère, estimant que les clercs devaient être d'office membres du Parti, raye les fêtes chrétiennes du calendrier des fêtes officielles, interdit des mouvements de jeunesse confessionnels, supprime des 
cours de religion dans les écoles, ferme des facultés de théologie catholique et protestante au sein de l'université nationale de Kinshasa, et se produit comme "prophète ", à l'image de Jésus : " Jésus est le prophète, Mobutu est notre prophète et notre libérateur, notre Messie. Le Christ est mort, il ne vit plus Lui. Il s'est dit Dieu. Mobutu n'est pas Dieu, il mourra (sic) aussi, mai il conduit son peuple vers une vie meilleure. Comment ne pas honorer, vénérer celui qui a fondé la nouvelle Église du Zaïre ? Notre Église est le Mouvement populaire de la Révolution. Son chef est Mobutu, nous le respectons comme on respecte le Pape. Notre Évangile est le mobutisme, le "Manifeste de la N'Sele"... Que vient faire le crucifix dans tous nos édifices publics? Il doit être remplacé par l'image de notre Messie. Et les militants auront à cœur de placer à ses cotés sa mère glorieuse, Mama Yemo, celle qui donna le jour à un tel fils. La sainte Vierge était aussi honorée comme mère du prophète Jésus » (p. 96-97).

9 Face à cette mégalomanie, l'Église répond: "Stanley et Léopold II ont disparu du paysage de notre capitale. Seuls les touristes le regrettent, provisoirement d'ailleurs, en attendant que nos architectes et artistes réaménagent ces sites. Nous voulons être chez nous, entre nous et résoudre ensemble nos problèmes selon ce que nous en penserons et déciderons. Nous voulons être authentiquement nous-mêmes et non plus nous laisser emporter dans une remorque conduite par d'autres [...]. De ce fait nous avons mis le doigt sur la question fondamentale, où se jouent en vérité notre grandeur et notre dignité de peuple libre. Mais cette entreprise est bien plus complexe qu'il n'y paraît à première vue. Allons-nous exhumer de la nuit du passé une "philosophie africaine originelle" qui n'a pu être, si du moins elle a un jour existé, que l'expression d'une situation et d'une vie sociale définitivement périmées? C'est dire que la découverte d'une telle conception de la vie ne saurait non plus être notre problème actuel ni nous aider à vivre dans le monde moderne. Notre monde n'étant plus celui de nos ancêtres, leur conception de la vie ne saurait non plus être la nôtre. Il est important que nous sachions cela clairement pour que nous ne perdions pas notre temps à bavarder d'une "négritude" un peu dépassée (sic) ... » (p. 92).

Le problème est que sur ce terrain de "philosophie africaine originelle ", Cl. Makiobo nous apprend que c'est l'Église qui, la première, a innové. En effet, il montre comment cette expression politique dans le champ religieux s'est formulée pour la première fois, de manière officielle, à travers une association d'anciens élèves catholiques dirigée par Malula, à l'époque encore abbé. En effet, l'association « Conscience africaine » que crée Malula en 1951, et qui publie un journal également dénommé Conscience africaine, sort, à l'occasion des commémorations de l'État indépendant du Congo, le 30 juin 1956, un numéro spécial sous le nom de "Manifeste de la conscience africaine » tiré à 10000 exemplaires et diffusé dans toutes les villes du Congo: ce fut le premier discours politique congolais. Ce manifeste jouera un rôle décisif dans la « prise de conscience du peuple congolais pour sa libération". Mais cette "prise de conscience du peuple congolais " se fait à la suite de celle que produisent des travaux des missionnaires coloniaux, parmi lesquels se détache la figure du célèbre père Placide Tempels qui explique à ses «frères européens » que les Bantous avaient une seule valeur : «la force vitale », qui organise toute leur pensée, tous les faits et gestes de la personne humaine. Ce système de pensée original pouvait donc, selon lui, servir de base à la fois à l'épanouissement africain et à l'essor du christianisme. Les travaux de Tempels s'inscrivent dans une lignée d'études linguistiques et ethnographiques qui conduisent «à la prise de conscience et à l'identité ethnique des peuples du Congo à une époque où un lourd préjugé défavorable pesait sur leur culture» (p. 39). Afin de poursuivre cette 
œuvre missionnaire, un département de philologie africaine est crée à l'université du Congo au lendemain de l'indépendance. Les missionnaires ont donc travaillé à la production de la conscience ethnique dans le contexte national crée par la colonisation, et cette conscience ethnique sera désormais inséparable de la conscience du peuple congolais produite par le manifeste de la conscience africaine de Malula et, plus tard, l'authenticité africaine de Mobutu. C'est encore en 1956, année de publication du "Manifeste de la conscience africaine", que les missionnaires vont pour la première fois se prononcer sur la propriété privée, le travail et sa rémunération, les associations professionnelles, les relations humaines et l'émancipation politique des Congolais. Les lettres pastorales, qui vont marquer la période postcoloniale, paraissent à cette date.

11 C'est encore sur le «terrain» de l'«authenticité africaine » que les Kimbanguistes soutiennent le projet « policico-idéologique » de Mobutu. Cl. Makiobo cite à ce sujet une circulaire adressée aux pasteurs et aux chrétiens kimbanguiste du Zaïre par son Éminence Diangenda, chef spirituel de l'Église kimbanguiste, par laquelle il leur demande "de se pénétrer de la philosophie de l'authenticité, de se soumettre à l'éducation permanente du parti et de rester toujours derrière le Guide (Mobutu) pour le triomphe de la révolution nationale » (p. 98). Pour l'auteur, «à cause, sans doute, de ses origines (le kimbanguisme est un mouvement messianique "authentiquement" africain. Son fondateur, Simon Kimbangou, prêchait l'avènement d'un Messie noir et d'un christianisme africain), l'Église kimbanguiste s'est laissée facilement intégrer dans le projet politique de Mobutu et ce dernier le reconnaît » (p. 97).

12 Cl. Makiobo analyse, également, la position de l'Église protestante: "Dès 1972, la hiérarchie nationale des Églises protestantes réunies au sein de l'Église du Christ au Zaïre (ECZ) a pris position en faveur du projet politico-idéologique de Mobutu » (p. 95). Mais l'auteur n'oublie pas, non plus, de signaler des dissensions au sein de l'épiscopat catholique et va jusqu'à faire valoir la force de l'argument matériel dans certaines positions de la hiérarchie catholique et rappelle les travaux de Jean-François Bayart ( Les Églises chrétiennes et la politique du ventre», in J.-F. Bayart, éd., Religion et modernité politique en Afrique noire. Dieu pour tous et chacun pour soi, Paris, Karthala, 1993) à propos de la "politique du ventre» des évêques qui reçoivent de Mobutu de « somptueux cadeaux » le jour de leur sacre ou à l'occasion de leur jubilé d'épiscopat.

Cl. Makiobo étudie également les « mouvements et associations à caractère spirituel qui ont en commun de partager la volonté d'innover le christianisme local et de transformer la société zaïroise » : il en cite une douzaine et limite son étude aux trois qui lui paraissent représentatifs, le mouvement des "Mamans catholiques», les «Bilenge ya Mwinda » et les « Kisito et Anuarite».

14 Ce livre est d'une lecture aisée : souci de clarté et de concision qui permet au lecteur intéressé par les rapports complexes entre religion et politique en Afrique de nourrir sa réflexion d'exemples particulièrement parlants. Car toute l'histoire de la contestation du pouvoir de Mobutu que raconte l'ouvrage est une histoire où religion et politique s'affrontent en brouillant en permanence les lignes de partage censées les séparer. 